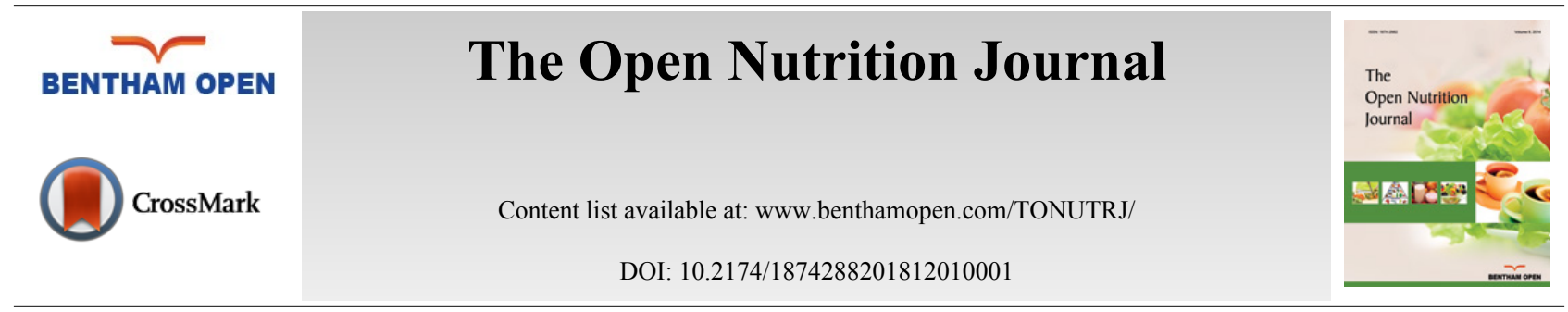

EDITORIAL

\title{
The Dilemma of Weight Cycling in Obesity: Is It Really a Health Risk Factor?
}

Obesity is a serious health problem, officially recognized by The American Medical Association (AMA) as a disease associated with several medical [1] and psychosocial comorbidities [2]. Its prevalence is increasing worldwide in adolescents and adults of both genders [3]. In recent decades, it has doubled in more than 70 countries, and is continuously increasing in most others. Particularly worrisome, however, is the finding that it has approximately tripled in children and young adults in developing and middle-income societies, for example Brazil and China. Indeed, the early onset of obesity is likely to translate into a high cumulative incidence of type 2 diabetes, cardiovascular disease and high rate of mortality [3].

Although in Western society this has prompted international guidelines to recommend a wide range of weight-loss interventions [4], most of these are known to be effective only in the short-term. In fact, weight regain is extremely common in patients with obesity who have intentionally lost weight, and almost $80 \%$ of patients are estimated to return to their baseline weight within five years of follow-up.

This phenomenon has been termed as "weight cycling" [5], and in the new millennium there has been great interest in studying its effects in humans [6]. Such research has however produced inconsistent findings, especially in terms of its adverse effects on health in obesity. Although there is a commonly held belief among the general population and some clinicians that weight cycling has a potential to predispose to future weight regain and provoke adverse effects on health [7], this has, by no means been demonstrated conclusively, and the effects of weight cycling on body composition, resting energy expenditure, and cardiovascular and metabolic status, for example, still remain to be explored $[8,9]$.

Furthermore, the majority of the existing scientific literature has been plagued by several important limitations, first and foremost a rather arbitrary definition of "weight cycling", which makes findings difficult to compare. Moreover, the cross-sectional design of most studies into weight cycling means that though they may indicate simple associations between "weight cycling" and some health parameter, they will never be able to provide solid information as to causal relationships. A part from this, another common methodological limitation is the reliance on self-reported weightcycling history, collected by means of questionnaires etc., which raises significant questions as to the accuracy of data (i.e., weight lost, weight regained, number of cycles etc.). Last but not least, some short-term studies (i.e. a few weeks) have investigated induced weight cycling with intentional weight regain, but this clearly cannot be a representative of the natural history of obesity, in which weight cycling occurs unintentionally and over a variable period of time (i.e. months or years) [8].

In some cases, misleading information regarding weight cycling has led clinicians to encourage patients to limit weight-loss attempts to prevent the adverse effects on health that they believe this phenomenon may produce [9]. Others are unsure as to how best to advise their patients. In light of this, The Open Nutrition Journal is seeking quality research on the topic, with the ultimate aim of providing a conclusive, evidence-based answer to the question: "Is weight cycling in obesity really a health risk factor?"

\section{REFERENCES}

[1] Eckel RH, Kahn R, Robertson RM, Rizza RA. Preventing cardiovascular disease and diabetes: A call to action from the American Diabetes Association and the American Heart Association. Circulation 2006; 113(25): 2943-6. 
[http://dx.doi.org/10.1161/CIRCULATIONAHA.106.176583] [PMID: 16801475]

[2] El Ghoch M, Calugi S, Dalle Grave R. The Effects of Low-Carbohydrate Diets on Psychosocial Outcomes in Obesity/Overweight: A Systematic Review of Randomized, Controlled Studies. Nutrients 2016; 8(7): 8. [PMID: 27367726]

[3] Afshin A, Forouzanfar MH, Reitsma MB, et al. GBD 2015 Obesity Collaborators. Health Effects of Overweight and Obesity in 195 Countries over 25 Years. N Engl J Med 2017; 377(1): 13-27.

[http://dx.doi.org/10.1056/NEJMoa1614362] [PMID: 28604169]

[4] Yumuk V, Tsigos C, Fried M, et al. Obesity Management Task Force of the European Association for the Study of Obesity. European Guidelines for Obesity Management in Adults. Obes Facts 2015; 8(6): 402-24.

[http://dx.doi.org/10.1159/000442721] [PMID: 26641646]

[5] National Task Force on the Prevention and Treatment of Obesity. Weight cycling. JAMA 1994; 272(15): 1196-202. [http://dx.doi.org/10.1001/jama.1994.03520150064038] [PMID: 7741844]

[6] Blackburn GL, Wilson GT, Kanders BS, et al. Weight cycling: the experience of human dieters. Am J Clin Nutr 1989; 49(5)(Suppl.): $1105-9$. [http://dx.doi.org/10.1093/ajcn/49.5.1105] [PMID: 2718940]

[7] Field AE, Byers T, Hunter DJ, et al. Weight cycling, weight gain, and risk of hypertension in women. Am J Epidemiol 1999; 150(6): 573-9. [http://dx.doi.org/10.1093/oxfordjournals.aje.a010055] [PMID: 10489996]

[8] Mackie GM, Samocha-Bonet D, Tam CS. Does weight cycling promote obesity and metabolic risk factors? Obes Res Clin Pract 2017; 11(2): 131-9. [http://dx.doi.org/10.1016/j.orcp.2016.10.284] [PMID: 27773644]

[9] Fothergill E, Guo J, Howard L, et al. Persistent metabolic adaptation 6 years after "The Biggest Loser" competition. Obesity (Silver Spring) 2016; 24(8): 1612-9.

[http://dx.doi.org/10.1002/oby.21538] [PMID: 27136388]

Marwan El Ghoch

Department of Eating and Weight Disorders

Villa Garda Hospital

Via Montebaldo

89, 37016 Garda (VR)

Italy

Tel: $+39-045-8103915$

Fax: +39-045-8102884

E-mail: marwan1979@hotmail.com

Member of the The Open Nutrition Journal editorial board

Simona Calugi

Department of Eating and Weight Disorders

Villa Garda Hospital

Via Montebaldo

89, 37016 Garda (VR)

Italy

Riccardo Dalle Grave

Department of Eating and Weight Disorders

Villa Garda Hospital

Via Montebaldo

89, 37016 Garda (VR)

Italy

C 2018 El Ghoch et al.

This is an open access article distributed under the terms of the Creative Commons Attribution 4.0 International Public License (CC-BY 4.0), a copy of which is available at: https://creativecommons.org/licenses/by/4.0/legalcode. This license permits unrestricted use, distribution, and reproduction in any medium, provided the original author and source are credited. 